\title{
Completely Positive Linear Maps in a Concept of Majorization on Certain Operator Algebras
}

By

Peter M. Alberti*

\begin{abstract}
Some concepts of Majorization in the state spaces of certain operator algebras are discussed and the interrelations among them are established. The main case considered deals with the class of non-finite AFD-factor vN-algebras.
\end{abstract}

\section{$\S 1$. Some Majorization Concepts on Operator Algebras}

Let $\mathfrak{A}$ be a unital $C^{*}$-algebra, with unitary group $\mathcal{U}(\mathfrak{U})$ and dual space $\mathfrak{Y} *$. Let $\mathfrak{B}\left(\mathfrak{A}^{*}\right)$ be the set of all bounded linear operators over $\mathfrak{A} *$ acting into $\mathfrak{2} *$ For $u \in \mathfrak{A}$, let us define $T_{u} \in \mathfrak{B}\left(\mathfrak{2} *^{*}\right)$ through the setting $\left(T_{u} \mu\right)(x)=\mu\left(u^{*} x u\right)=$ $\mu^{u}(x)$, for all $x \in \mathfrak{A}, \quad \mu \in \mathfrak{U}^{*}$. We put $\mathcal{C}_{u}(\mathfrak{U})=$ point- $w_{-} *$ closure of conv $\left\{T_{v}: v \in \mathcal{U}(\mathfrak{A})\right\}$, where the point- $w^{*}$-topology refers to the system of semi-norms $p_{\nu, a}$ over $\mathfrak{B}(\mathfrak{A} *)$ given by $p_{\nu, a}(T)=|(T \nu)(a)|, \nu \in \mathfrak{Y} *, a \in \mathfrak{A}$. Then, by wellknown facts, $\mathcal{C}_{u}(\mathfrak{U})$ is a convex and compact subset of $\mathfrak{B}\left(\mathfrak{U}^{*}\right)$ with respect to the topology mentioned. Suppose, $S(\mathfrak{U})$ denotes the $w^{*}$-compact convex set of states over $\mathfrak{A}$. Throughout this paper we let $n$ be a fixed natural number. Whenever $\nu_{1}, \cdots, \nu_{n} \in S(\mathfrak{U})$ are given, the finite sequence $\left\{\nu_{1}, \cdots, \nu_{n}\right\} \subset S(\mathfrak{U})$ will be referred to as a $n$-tuple of states, which will be abbreviated by $\{\nu\}$. In all what follows we shall have to work in the set of all $n$-tuples of states over $\mathfrak{A}$. Within this set of all $n$-tuples of states we are going to introduce a preordering relation $\gg_{u}$. A preordering in our context is supposed to mean a reflexive and transitive relation: $\{\omega\} \gg_{u}\{\omega\}, \forall\{\omega\}$, and $\{\omega\} \gg_{u}\{\nu\}$ together with $\{\nu\} \gg_{u}\{\sigma\}$ implies always $\{\omega\} \gg_{u}\{\sigma\}$.

Communicated by H. Araki, June 11, 1986.

* Sektion Mathematik und Naturwissenschaftlich-Theoretisches Zentrum (NTZ), Karl-MarxUniversität Leipzig, DDR-7010 Leipzig. 
Definition 1. For n-tuples $\{\omega\},\{\sigma\} \subset S(\mathfrak{V})$ we let $\{\omega\} \gg_{u}\{\sigma\}$ mean that $\omega_{k}=\Phi\left(\sigma_{k}\right), \forall k$, with $\Phi \in \mathcal{C}_{u}(\mathfrak{U})$.

In this situation, let us say that " $\{\omega\}$ is more unitarily mixed than $\{\sigma\}$ " or " $\{\omega\}$ arises from $\{\sigma\}$ through unitary mixing".

It is easy to see that the concept of unitary mixing for $n$-tuples of states as introduced above is a straightforward extension of the concept of $G$-majorization as given in $[1$, Chap. $14, \mathbb{B}]$. Namely, what we are doing is to consider the $n$-fold direct product $C^{*}$-algebra $\mathfrak{U}_{n}$ (i.e. the set of $n$-tuples $\{a\}=\left\{a_{1}, \cdots, a_{n}\right\}$ of elements $\left.a_{j} \in \mathfrak{U}\right)$. Then, the set of all $n$-tuples of states over $\mathfrak{A}$ can be interpreted as a convex, $w^{*}$-compact subset of elements of $\mathfrak{U}_{n+}^{*}$ in an obvious way. Within $\mathfrak{B}\left(\mathfrak{U}_{n}^{*}\right)$, let us define a group $G$ of transformations by $G=\left\{T \in \mathfrak{B}\left(\mathfrak{U}_{n}^{*}\right)\right.$ : $\left.T=\left\{T_{v}, T_{v}, \cdots, T_{v}\right\}, v \in \mathcal{U}(\mathfrak{U})\right\}$, where the action of $S=\left\{S_{1}, \cdots, S_{n}\right\}$ on the $n$-tuple $\{\nu\}$ is given via $S(\{\nu\})=\left\{S_{1}\left(\nu_{1}\right), \cdots, S_{n}\left(\nu_{n}\right)\right\}$. Then, the relation $\{\omega\} \gg_{u}\{\sigma\}$ between the $n$-tuples mentioned is equivalent to saying that the $\{\omega\}$-element of $\mathfrak{U}_{n+}^{*}$ is in the $w^{*}$-closed convex hull of the orbit of the $\{\sigma\}$-element under the action of the group $G$. The relations to the concept of $G$-majorization should be clear now by remembering that the original concept as represented in [1] can also be interpreted in terms of $C^{*}$-algebras. Indeed, $\mathbb{R}^{N}$ might be thought of as the hermitian part of $\mathfrak{2} *$ when $\mathfrak{Q}=\mathbb{C}^{N}$ is chosen, cf. Section 2, Example 1, and $n=1$ is considered.

In the following, let us introduce and discuss another preordering relation $\gg$ over the set of $n$-tuples of states on $\mathfrak{U}$. A linear map $\Phi: \mathfrak{U}^{*} \rightarrow \mathfrak{U}^{*}$ is referred to as a c.p.-stochastic linear map, and the set of all these mappings be $S T\left(\mathfrak{U}^{*}\right)$, if $\Phi$ carries states into states and the adjoint $\Phi^{\#}$ is a completely positive, unital linear transformation over the second dual $\mathfrak{2} * *$ (for generalities on $C^{*}$ - and $W^{*}$-algebras the reader is referred to the standard textbooks, e.g. [2],

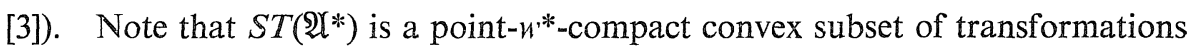
of $\mathfrak{B}\left(\mathfrak{U}^{*}\right)$, which also forms a semigroup and contains the identity map.

Definition 2. For n-tuples $\{\omega\},\{\sigma\} \subset S(\mathfrak{Y})$ put $\{\omega\} \gg\{\sigma\} \Leftrightarrow \Xi \Phi \in \mathbb{S} T\left(\mathfrak{\mathscr { U }} *^{*}\right)$ with $\omega_{k}=\Phi\left(\sigma_{k}\right), \forall k$.

In case we are given a vN-algebra $\mathfrak{A}=\mathfrak{M}$ acting over some Hilbert-space $\mathfrak{S}$, a third preordering relation $\gg_{*}$ will be of further interest. Let $\mathfrak{M E}_{*}$ be the predual of $\mathfrak{M}$, and $S_{0}(\mathfrak{M})$ the set of normal states over $\mathfrak{M}$. Then, a linear map $\psi: \mathfrak{M}_{*} \rightarrow \mathfrak{M}_{*}$ is said to be c.p.-stochastic over $\mathfrak{M}_{*}$, and we collect all these mappings into the set $S T\left(\mathfrak{M E}_{*}\right)$, if the adjoint $\psi^{*}$ over $\mathfrak{M}$ is completely positive and unital. Note that in the latter case $\psi^{\#}$ is a normal positive linear map in 
the usual sense. We shall denote by $C P_{1}(\mathfrak{M})$ and $C P_{1}^{0}(\mathfrak{M})$ the set of unital c.p.linear maps over $\mathfrak{M}$ and the normal unital c.p.-mappings over $\mathfrak{M} \&$, respectively.

$\mathbb{D e f i n a i t i o n ~ 3 . ~ F o r ~} n$-tuples $\{\omega\},\{\sigma\} \subset S_{0}(\mathfrak{M})$ of normal states we define $\{\omega\} \gg *\{\sigma\} \Leftrightarrow \exists \Phi \in S T\left(\mathfrak{M}_{*}\right)$ with $\omega_{k}=\Phi\left(\sigma_{k}\right), \forall k$.

Note that $\gg_{*}$ is a preordering relation since $S T\left(\mathfrak{M}_{*}\right)$ is a semigroup in $\mathfrak{B}\left(\mathfrak{M}_{*}\right)$ containing the identity map.

Remark. Whereas by Def. 1 the concept of $G$-majorization has been extended, Def. 2 and Def. 3 relate to the extension of the concept of a stochastic matrix or stochastic operator from the commutative case (either $\mathfrak{M}=\mathbb{C}^{N}$, or more generally $\mathfrak{M}=L^{\infty}(\Omega, \mu), \mathfrak{M}_{*}=L^{1}(\Omega, \mu)$, with some localizable measure space $(\Omega, \mu)$ ) to the general non-commutative situation of a unital $C^{*}$-algebra or $W^{*}$-algebra, cf. [4] and references quoted there. Note that $\{\omega\} \gg *\{\sigma\}$ is equivalent with the existence of $T \in C P_{1}^{0}(\mathfrak{M})$ such that $\omega_{k}=\sigma_{k} \circ T, \forall k$. The convex semigroups $C P_{1}(\mathfrak{M})$ and $C P_{1}^{0}\left(\mathfrak{M}_{\mathcal{E}}\right)$ both contain the identity map and extend to the non-commutative situation the notions of Markovian operators and normal Markovian operators, respectively, which are known from the abelian cases.

Majorization theory as one of its basic goals requires to establish functional characterizations of the preordering relations concerned. By this the following is meant. Assume a preordering $\ggg$ on our set of $n$-tuples of states (resp. normal states) is given. A realvalued function $F$ over the set of $n$-tuples of states will be said to be isotonic with respect to the preordering in question, whenever the implication $\{\omega\} \ggg\{\sigma\} \Rightarrow F(\{\omega\}) \geq F(\{\sigma\})$ takes place, and $F$ is said to be $\gg$-anti-isotonic, if the implication $\{\omega\} 》\{\sigma\} \Rightarrow F(\{\omega\}) \leq F(\{\sigma\})$ is always true. Aiming at a functional characterization of $\gg$ then means to meet two lines:

(1) Impose conditions on a realvalued function $F$ which imply that $F$ is isotonic (resp. anti-isotonic) and which do not read in terms of the very definition of the preordering.

(2) Extract a sufficient system ( $\widetilde{\Upsilon}, 》)$ of isotonic (resp. anti-isotonic) functions such that always $F(\{\omega\}) \geq F(\{\sigma\}), \forall F \in \mathfrak{Y}, \Rightarrow\{\omega\} \gg\{\sigma\}$ is true (resp. with $\geq$ substituted by $\leq$ in the anti-isotonic case).

Clearly, a theory of majorization tacitely confines to cases where such a sufficient system of functions exists and reflects the non-trivial character of the preordering considered. Besides this fact, however, there might be a variety of sufficient systems which might differ from each other. It is of an obvious use to keep 
the sufficient systems as "small" as possible. Speaking in words of (1) one is tempted to look for conditions on the functions which are as "stringent" as possible when imposed on.

We shall try to meet this task of a functional characterization in case of $\gg_{*}$ in the set of normal states over a properly infinite, approximately finitedimensional factor on a separable Hilbert-space.

\section{§2. Classical Examples}

In all what follows we shall strictly adhere on interpreting the majorization concepts in an algebraic language, even if these concepts do not refer to this context in their original formulation.

Example 1. Let $\mathfrak{A}$ be a finite-dimensional (say $N$-dimensional) abelian $C^{*}$-algebra, and let Aut $\mathfrak{A}$ be the full group of linear *automorphisms over $\mathfrak{A}$. Define $G$ as a group of actions of Aut $\mathfrak{A}$ on $\mathfrak{Y} *$ by defining $T_{g} \in G$ through $\left(T_{g} \nu\right)(x)=\nu(g(x)), \forall x \in \mathfrak{A}, \nu \in \mathfrak{U} *$, for each $g \in$ Aut $\mathfrak{A}$. An element $\nu \in S(\mathfrak{U})$ can be characterized by the values it takes on the atoms (call them spectral values). Let $\nu^{1} \geq \nu^{2} \geq \cdots \geq \nu^{N}$ be the decreasingly ordered sequence of these spectral values (one has to take care to the multiplicity of the spectral values). Let $\omega, \sigma$ be states, and let $\omega \gg_{G} \sigma$ mean that the state $\omega$ is in the convex hull of the orbit of $\sigma$ under $G$.

This then will give the starting point for one of the classical examples majorization theory deals with: $\omega \gg_{G} \sigma$ is equivalent with the following conditions,

$$
\begin{array}{ll}
\Leftrightarrow & \sum_{j \leq k} \omega^{j} \leq \sum_{j \leq k} \sigma^{j}, \forall k ; \\
\Leftrightarrow & F(\omega) \leq F(\sigma)
\end{array}
$$

for any quasi-convex (see [1]), $G$-invariant function $F$ over $S(\mathfrak{A})$;

$$
\Leftrightarrow \quad \sum_{j} f\left(\omega^{j}\right) \leq \sum_{j} f\left(\sigma^{j}\right)
$$

for any convex, continuous function $f$ on $[0,1]$.

In this example, in which we refer to the fundamental result of the theory of inequalities in [5] (Muirhead's Theorem), Theorems of Rado and Birkhoff (see [1], [4]), we have given three different functional characterizations of the $G$-majorization in question in terms of anti-isotonic functions. Whereas the system of functions in (2-2) certainly is the largest of the given three, the one of $(2-1)$ is the smallest under comparison (it contains $n-1$ functions, the $n$-th 
being constant on $S(\mathfrak{U})$ ). This provides a good example to illustrate the discussion below (1), (2) in the previous section.

In the next example we refer to Def. 1 with $n=1$, and $\mathfrak{U}$ is an arbitrary $\mathrm{vN}$-algebra (we omit the parantheses in case of 1-tuples).

Example 2. For states $\omega, \sigma \in S(\mathfrak{U}), \omega \gg_{u} \sigma$ is equivalent with the following two conditions:

$$
\Leftrightarrow \quad F(\omega) \leq F(\sigma)
$$

for every quasi-convex, $w^{*}$-lower semicontinuous, unitarily invariant function $\mathbb{F}$ over $S(\mathfrak{U})$;

$$
\Leftrightarrow
$$

$$
\sup _{u \in \mathcal{U}(\mathfrak{R})} \omega^{u}(p) \leq \sup _{u \in \mathcal{U}(\mathfrak{R})} \sigma^{u}(p)
$$

for each of the orthoprojection of $\mathfrak{A}, p \in \mathfrak{U}$.

This example (unitary mixing on $\mathrm{vN}$-algebras) has been investigated extensively by several authors, and is represented in [6], cf. the list of references there. The original concept is due to A. Uhlmann and refers to the case of $\mathfrak{A}=\mathbb{M}_{N}$, the full algebra of complex $N \times N$-matrices, see [7]. In connection with its applications in Statistical Physics, the whole subject for density operators is referred to as "Uhlmann's Theory", and "order structure of states on $W^{*}$. algebras" in the general case, respectively, see [8], [9]. Once more again, the structure of the example reflects the significance of the discussion at the end of Section 1.

Note that because of the Murray-von Neumann theory of factors a sufficient set of functions which figures in $(2-5)$ in case of factors over a separable Hilbert-space can be parametrized conveniently by the relative dimension $\tau$.

Indeed, let us define functions $\left\{e_{\tau}: \tau \in \Delta\right\}$ over $S(\mathfrak{U})$, with the range $\Delta$ of the relative dimension given by $\Delta=\{0,1, \cdots, N\}$ for type $\mathbb{I}_{N}, \Delta=\mathbb{N} \cup\{0, \infty\}$ for type $\mathbb{I}_{\infty}, \Delta=[0,1]$ for type $\mathbb{I}_{1}, \Delta=[0, \infty]$ for type $\mathbb{I}_{\infty}$, and $\Delta=\{0, \infty\}$ in the remaining type III cases of factors. The function $e_{\tau}$ reads $e_{\tau}(\nu)=\sup _{u \in \mathcal{U}(\mathfrak{X})} \nu^{u}(p)$, for an orthoprojection $p$ with relative dimension $\tau \in \Delta, \forall \nu$. Then, (2-5) is equivalent to

$$
\Leftrightarrow \quad e_{\tau}(\omega) \leq e_{\tau}(\sigma), \quad \forall \tau \in \Delta .
$$

Hence, $\left\{e_{\tau}: \tau \in \Delta\right\}$ is a set of unitarily invariant, convex functions over $S(\mathfrak{U})$ which forms a sufficient system of $\gg_{u}$-anti-isotonic functions in case of a factor, by (2-6). 
Example 3. Suppose $\mathfrak{M}$ is a $\mathrm{vN}$-factor of finite type. Let us look on the case $n=2$ (the case of tuples) and restrict $\gg_{*}$ (see Def. 3 ) to all pairs of normal states the second state of which is the canonical trace-state $\tau$. Then

$$
\{\omega, \tau\} \gg_{*}\{\sigma, \tau\} \Leftrightarrow \omega \gg_{u} \sigma
$$

in the sense of Example 2. Therefore, the sufficient systems of anti-isotonic functions (2-4), (2-5) and (2-6) characterize $\gg_{u}$ as well as $\gg_{*}$ on the subset considered (see [6]).

Example 4. Let $\mathfrak{M}=L^{\infty}([0,1])$ (the unit interval with Lebesgue measure), and suppose $n=2$. Then, for normal states $\omega_{1}, \omega_{2}, \sigma_{1}, \sigma_{2}$ (which correspond to probability distributions)

$$
\left\{\omega_{1}, \omega_{2}\right\} \gg_{*}\left\{\sigma_{1}, \sigma_{2}\right\} \Leftrightarrow\left\|\omega_{1}-t \omega_{2}\right\|_{1} \leq\left\|\sigma_{1}-t \sigma_{2}\right\|_{1}, \quad \forall t \in \mathbb{R}_{+}
$$

This has been proved in [10]. Note that the assertion remains true also in the non-abelian case $\mathfrak{M}=M_{2}$, see [11], [6].

The next example to be considered is a very instructive one and deals with the general $n$-tuple situation over an abelian $\mathrm{vN}$-algebra.

Example 5. Let $\mathfrak{M}$ be a commutative $W^{*}$-algebra. Then, $\mathfrak{M}$ is isomorphic with $L^{\infty}(\Omega, \mu)$ for some suitable localizable measure space $(\Omega, \mu)$. Let $\{\omega\},\{\sigma\} \subset S_{0}(\mathfrak{M})$ be $n$-tuples of normal states ovre $\mathfrak{M}$. Considering $\mathfrak{M} \simeq$ $L^{\infty}(\Omega, \mu)$, a normal state $\nu$ corresponds to a probability distribution

$$
\nu \in L^{1}(\Omega, \mu), \quad \text { and } \quad \nu(x)=\int_{\Omega} \nu(t) x(t) d \mu(t), \quad \text { for } \quad x \in L^{\infty}(\Omega, \mu) .
$$

With all this notations adopted, we have:

$$
\begin{aligned}
& \{\omega\} \gg *\{\sigma\} \Leftrightarrow\{\omega\} \gg\{\sigma\} \Leftrightarrow \\
& \int_{\Omega} \omega_{n}(t) G\left(\omega_{1}(t) / \omega_{n}(t), \cdots, \omega_{n-1}(t) / \omega_{n}(t)\right) d \mu(t) \geq \int_{\Omega} \sigma_{n}(t) G\left(\sigma_{1}(t) / \sigma_{n}(t), \cdots\right. \\
& \left.\sigma_{n-1}(t) / \sigma_{n}(t)\right) d \mu(t)
\end{aligned}
$$

for every non-negative, concave function $G$ over $\mathbb{R}_{+}^{n-1}$.

This means, the homogeneous and superadditive functionals

$$
H_{G}\left(\nu_{1}, \cdots, \nu_{n}\right)=\int_{\Omega} \nu_{n} G\left(\nu_{1} / \nu_{n}, \cdots, \nu_{n-1} / \nu_{n}\right) d \mu
$$

over the convex set of $n$-tuples of normal states of $\mathfrak{M}$ provide a sufficient system 
of isotonic functions in both the $\gg$ and the $\gg *$ case. But note that (2-9) has to be defined with some care (at the boundary of the cone $\mathbb{R}_{+}^{n-1}$ one has to adopt some convention concerring the function under the integral; we omit the details, but refer to [13]).

Note that for $n=2, \Omega=[0,1], \mu$-Lebesgue measure, the given equivalences mean that $(2-8) \Leftrightarrow(2-7)$, which fact is known since [10]. Basic results relating the equivalences given have been derived in [12] and considered systematically in [14], especially in the case where the measure space is finite and $\nu_{n}=\omega_{n}=$ $\sigma_{n}=\mu(\Omega)^{-1}$, which means doubly stochasticity of all transformations involved. The complete set of equivalences in the general case is shown in [13]. Obviously, the example reads in the classical tradition of majorization theory, theory of stochastic and doubly stochastic transformations on $\mathbb{L}^{1}$-spaces, and so has much to do with rearrangement considerations, see references given in [13], and [15] for related topics.

Note that the functions (2-9) form only one specific sufficient system of isotonic functions. A sufficient system of anti-isotonic functions is given by

$$
S_{\left\{x_{1}, \cdots x_{n}\right\}}\left(\nu_{1}, \cdots, \nu_{n}\right)=\int_{\Omega}\left\|\nu_{1}(l) x_{1}+\cdots+\nu_{n}(t) x_{n}\right\|_{\infty} d \mu(t)
$$

for any $n$-tuple $\{x\} \subset L^{\infty}(\Omega, \mu)_{+2}$ see [13], [14].

\section{§3. Notations, the Problem of This Paper}

The objective of this paper is to show the intimate relations among the preorderings $\gg, \gg_{*}, \gg_{u}$ introduced in Section 1 . In this connection, we will find that such relations can only exist in cases of certain classes of noncommutative vN-algebras and related to them $\mathbb{C}^{*}$-algebras. That means, we restrict the subject of our investigations from the very beginning to those structures which seem most promising. In line with the examples of the previous section, and following the discussion of majorization concepts in Section 1 , what will be done is to construct sufficient systems of isotonic resp. anti-isotonic functions, and to investigate their properties.

The central class of algebras under consideration consists of the approximctely finite-dimensional factors (AFD-factors) over some separable Hilbertspace $\mathfrak{S}$. Let $\mathfrak{M}$ be such a factor. Then, there exists an increasing sequence

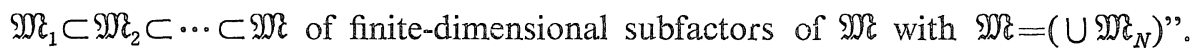
Let $\mathfrak{U}_{0}=\cup \mathfrak{M R}_{N}$, and define $\mathfrak{U}=$ norm closure $\mathfrak{U}_{0}$. The unital $\mathbb{C}^{*}$-algebra $\mathfrak{U}$ is referred to as a generating U.H.F.- $C^{*}$-subalgebra of $\mathfrak{M}$ (U.H.F. means 
uniformly hyperfinite). AFD-factors can be characterized by several conditions all of which are equivalent, see [16], and [17] for a new and instructive proof of these facts. The non-finite AFD-factors - these are all AFD-factors with exclusion of the hyperfinite factor (which is the type $\mathrm{I}_{1}$ case) - will be of special interest in our investigations. Let us call a vN-algebra $\mathfrak{N}$ over $\mathfrak{S}$ admissible if there is a non-finite AFD-factor $\mathfrak{M}$ over $\mathfrak{S}$ such that $\mathfrak{R} \subset \mathfrak{M}$ and a normal conditional expectation $E$ projecting from $\mathfrak{M}$ onto $\mathfrak{N}$ exists. Note that both the finite dimensional factor case and the hyperfinite factor case are admissible in our sense. Of course, there are much more admissible cases than the mentioned ones.

\section{§4. An Account of Basic Results}

Let $\mathfrak{M}$ be a AFD-factor over the separable Hilbert-space $\mathfrak{E}$, and suppose $\mathfrak{A}$ is a generating U.H.F.- $C^{*}$-subalgebra of $\mathfrak{M}$. For $n$-tuples of normal states $\{\nu\} \subset S_{0}(\mathfrak{M})$ and positive elements $\{x\} \subset \mathfrak{M}_{+}$let us define

$$
\begin{aligned}
& K_{n}(\{\nu\},\{x\})=\sup _{T}\left\{\sum \nu_{k} \circ T\left(x_{k}\right): T \in C P_{1}^{0}(\mathfrak{M})\right\} \\
& K_{n}^{\prime}(\{\nu\},\{x\})=\sup _{u}\left\{\sum \nu_{k}\left(u^{*} x_{k} u\right): u \in \mathcal{U}(\mathfrak{M})\right\}, \\
& K_{n}(\mathfrak{A} ;\{\rho\},\{y\})=\sup _{u}\left\{\sum \rho_{k}\left(u^{*} y_{k} u\right): u \in \mathcal{U}(\mathfrak{A})\right\}
\end{aligned}
$$

if $\{\rho\} \subset S(\mathfrak{U})$ and $\{y\} \subset \mathfrak{U}_{+}$. In [18], the following two results have been derived for AFD-factors.

Proposition 1. To every generating $\mathfrak{U}$, there exists a net $S_{\lambda}: \mathfrak{M} \rightarrow \mathfrak{A}_{0}$ of completely positive, $\sigma$-weakly continuous linear mappings into $\mathfrak{U}_{0}$ such that

$$
K_{n}(\{\nu\},\{x\})=\lim _{\lambda} K_{n}\left(\{\nu\},\left\{S_{\lambda}(x)\right\}\right)=\sup _{\lambda} K_{n}\left(\{\nu\},\left\{S_{\lambda}(x)\right\}\right)
$$

for each n-tuple of normal states and any $\{x\} \subset \mathfrak{M}_{+}$.

Remark. The proof uses the condition "semidiscreteness" which holds for AFD-factors. The right hand side equality in (4-4) is a consequence of the proof in [18, Lemma 2].

Proposition 2. For a non-finite AFD-factor Me one has

$$
K_{n}(\{\nu\},\{y\})=K_{n}^{\prime}(\{\nu\},\{y\})=K_{n}\left(\mathscr{A} ;\left\{\nu_{/ 22}\right\},\{y\}\right)
$$

for all $\{y\} \subset \mathfrak{U}_{+}$and every n-tuple $\{\nu\} \subset S_{0}(\mathfrak{M})$ of normal states $\left(\nu_{12 \mathbb{X}}\right.$ indicates the restriction of $\nu$ onto $\mathfrak{\Re})$. 
These two facts together give the key for a proof of the following basic result.

Theorem 1. Let $\mathfrak{M e}$ be a non-finite AFD-factor, and $\mathfrak{U}$ be an arbitrary generating U.H.F.- $C^{*}$-subalgebra. Assume $\{\omega\},\{\sigma\}$ are n-tuples of normal states over $\mathfrak{M}$. The following conditions are equivalent to each other:

(1) $\left\{\omega_{/ 2\}}\right\} \gg_{u}\left\{\sigma_{/ 22}\right\} \quad$ (cf. Def. 1);

(2) $\{\omega\} \gg\{\sigma\} \quad$ (cf. Def. 2);

(3) $\{\omega\} \gg_{*}\{\sigma\} \quad$ (cf. Def. 3);

(4) $K_{n}(\{\omega\},\{x\}) \leq K_{n}(\{\sigma\},\{x\}), \forall\{x\} \subset \mathfrak{M}_{+}$;

(5) $K_{n}^{\prime}(\{\omega\},\{x\}) \leq K_{n}^{\prime}(\{\sigma\},\{x\}), \forall\{x\} \subset \mathscr{N}_{0+} ;($ !)

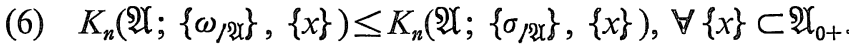

For a detailed proof see [18]. The remarkable feature in the result lies in the fact that all problems around $\gg$ or $\gg_{*}$ between normal states may be handled equivalently as unitary mixing problems on the subset $\left.S_{0}(\mathfrak{M})\right)_{/ 2}$ of the state space of an arbitrarily given generating U.H.F.- $C^{*}$-subalgebra $\mathfrak{A}$. Moreover, the system of functions

$$
\mathfrak{S}_{0}=\left\{K_{n}(\{\cdot\},\{x\}):\{x\} \subset \mathfrak{M}_{+}\right\}, \quad \mathfrak{S}^{\prime}(\mathfrak{U})=\left\{K_{n}^{\prime}(\{\cdot\},\{x\}):\{x\} \subset \mathfrak{A}_{0+}\right\},
$$

$\mathfrak{\Im}(\mathfrak{U})=\left\{K_{n}(\mathfrak{A} ;\{\circ, \mathfrak{A}\},\{x\}):\{x\} \subset \mathfrak{U}_{0+}\right\}$ provide us with sufficient systems of anti-isotonic functions in the sense discussed in Section 1 in either case. By (4-1)-(4-3) and our Propositions, it is easy to see that $\mathfrak{\Im}_{0} \supset \mathfrak{\Im}^{\prime}(\mathfrak{U})$, and $\mathfrak{\Im}^{\prime}(\mathfrak{U})=$ $\mathfrak{\Im}(\mathfrak{U})$ for every generating U.H.F.- $C^{*}$-algebra $\mathfrak{A}$ holds, provided $\mathfrak{M}$ is properly infinite (i.e. $\mathfrak{M}$ is non-finite, since we are in the factor case).

\section{§. Properties of Some Convex and Concave Functions}

Let us adopt all the notations of the previous sections. Throughout, assume $\mathfrak{M}$ is a non-finite AFD-factor, with an arbitrarily chosen, but throughout fixed, generating U.H.F.-C*-subalgebra $\mathfrak{A}$, and $\mathfrak{A}_{0}=\cup \mathfrak{M R}_{N}$. We are going to introduce some further notations. Let $\{x\} \subset \mathfrak{M}$ be a $n$-tuple of elements of $\mathfrak{M}$. $\{x\}$ will be referred to as an algebraically finite-dimensional system, a.f.d.-system for short, if there is a finite-dimensional subfactor $F$ of $\mathfrak{M}$ such that $\{x\} \subset F \subset$ $\mathfrak{M}$. By $\Lambda_{n}(\mathfrak{M})$ let us denote the set of all $n$-tuples in $\mathfrak{M}$ which form a.f.d.systems, and $A_{n}(\mathfrak{M})_{+}$be the subset which consists of $n$-tuples of positive "elements. Let us introduce two further sets of functions:

$$
\begin{aligned}
\mathfrak{\Im}_{n}^{+}(\mathfrak{M})=\left\{f \in \mathbb{R}_{+}^{S_{0}(\mathfrak{M}) \times \cdots \times S_{0}(\mathfrak{M})}: f(\{\omega\})=\sup _{u \in \mathcal{U}(\mathfrak{M})} \sum \omega_{k}^{u}\left(x_{k}\right),\right. \\
\left.\quad \text { for some }\{x\} \in \Lambda_{n}(\mathfrak{M})_{+}\right\}
\end{aligned}
$$




$$
\begin{aligned}
& \mathfrak{\Im}_{n}^{-}(\mathfrak{M})=\left\{f \in \mathbb{R}_{+}^{S_{0}(\mathfrak{M}) \times \cdots \times S_{0}(\mathfrak{M})}: f(\{\omega\})=\inf _{u \in \mathcal{U}(\mathfrak{M})} \sum \omega_{k}^{u}\left(x_{k}\right),\right. \\
& \text { for some } \left.\{x\} \in \Lambda_{n}(\mathfrak{M})_{+}\right\}
\end{aligned}
$$

Both sets of functions are intimately related families. Namely, let $f \in \mathfrak{S}_{n}^{+}(\mathfrak{M} \mathcal{R})$ be generated by $\{x\} \in \Lambda_{n}(\mathfrak{M})_{+}$, and $g \in \mathfrak{S}_{n}^{-}(\mathfrak{M})$ be generated by the a.f.d.system $\{y\}$. Then, there are constants $\alpha, \beta \in \mathbb{R}_{+}$and $g^{\prime} \in \mathfrak{\Im}_{n}^{-}(\mathfrak{M}), f^{\prime} \in \mathfrak{S}_{n}^{+}(\mathfrak{M})$ such that

$$
\begin{aligned}
& f=\alpha-g^{\prime}, \\
& g=\beta-f^{\prime}
\end{aligned}
$$

In fact, let $\alpha=\sum\left\|x_{k}\right\|$, and $g^{\prime}$ be the element of $\mathfrak{\Im}_{n}^{-}(\mathfrak{M})$ generated by the a.f.d.system $\left\{y^{\prime}\right\}$ with $y_{k}^{\prime}=\left\|x_{k}\right\| 1-x_{k}, \forall k$; for $\beta$ take $\beta=\sum\left\|y_{k}\right\|$, and let $f^{\prime}$ be the element of $\mathfrak{\Im}_{n}^{+}(\mathfrak{M})$ generated by the a.f.d.-system $\left\{x^{\prime}\right\}$ given through $x_{k}^{\prime}=$ $\left\|y_{k}\right\| 1-y_{k}, \forall k$. Then, a verification of (5-1) and (5-2) is a matter of easy inserting, hence the details are omitted. Owing to (5-1) and (5-2), the properties of the two systems of functions are dual to each other. We shall investigate the basic properties of the family $\mathfrak{S}_{n}^{+}(\mathfrak{M})$.

Lemma $\mathbb{1}_{\text {。 }}$ Let $f \in \mathfrak{S}_{n}^{+}(\mathfrak{M} \mathfrak{)})$. There is $\{y\} \subset \mathfrak{A}_{0+}$ such thai

$$
f(\{\omega\})=K_{n}^{\prime}(\{\omega\},\{y\}), \forall\{\omega\} \subset S_{0}(\mathfrak{M}) .
$$

Proof. Let $f$ be generated by $\{x\} \in \Lambda_{n}(\mathfrak{M})_{+}$. Hence, there is a finitedimensional subfactor $F$ of $\mathfrak{M}$ which contains the a.f.d.-system $\{x\}$. Let us chose the subscript $N$ such that $\operatorname{dim} \mathfrak{M C}_{N} \geq \operatorname{dim} F$ (dim means the relative dimension of the subfactors under discussion). We are going to show that $v \in \mathfrak{B}(\mathfrak{S})$, with $v^{*} v=\mathbb{1}$, exists such that $v^{*} \mathfrak{M}_{N} v=F$. In fact, we find unitaries $u_{1}, u_{2} \in \mathcal{U}(\mathfrak{S})$ with the property that $u_{1} \mathfrak{M}_{N} u_{1}^{*}=\mathfrak{B}\left(\mathfrak{E}_{1}\right) \otimes 1, u_{2} F u_{2}^{*}=\mathfrak{B}\left(\mathfrak{S}_{1}^{\prime}\right) \otimes 1$, with Hilbertspaces $\mathfrak{S}_{1}, \mathfrak{S}_{1}^{\prime}, \mathfrak{S}_{2}, \mathfrak{S}_{2}^{\prime}$ such that $\mathfrak{S}_{2} \mathfrak{S}_{1} \otimes \mathfrak{S}_{2}, \mathfrak{L}_{=}=\mathfrak{S}_{1}^{\prime} \otimes \mathfrak{S}_{2}^{\prime}$, and $\operatorname{dim} \mathfrak{L}_{1}=\operatorname{dim} \mathfrak{M R}_{N}$, $\operatorname{dim} \mathfrak{S}_{1}^{\prime}=\operatorname{dim} F$. Let $p^{\prime} \in \mathfrak{B}\left(\mathfrak{S}_{1}\right)$ be an orthoprojection with $\operatorname{dim} p^{\prime}=\operatorname{dim} \mathfrak{S}_{1}^{\prime}$. Let $\left\{\Psi_{k}\right\},\left\{\Psi_{k}^{\prime}\right\}$ be complete orthonormal systems of $\mathfrak{S}_{2}, \mathfrak{S}_{2}^{\prime}$, respectively, and $\left\{\varphi_{j}\right\},\left\{\varphi_{j}^{\prime}\right\}$ complete orthonormal systems in $p^{\prime} \mathfrak{E}_{1}, \mathfrak{S}_{1}^{\prime}$, respectively.

We might define an isometry $w \in \mathfrak{B}(\mathfrak{S})$ through the settings $w \varphi_{k}^{\prime} \otimes \Psi_{j}^{\prime}=$ $\varphi_{k} \otimes \Psi_{j}, k=1,2, \cdots, \operatorname{dim} F, j \in \mathbb{N}$. Then, $w^{*} w=1, \quad w w^{*}=p^{\prime} \otimes 1$. Moreover, one easily recognizes that $w^{\mathfrak{B}}\left(\mathfrak{S}_{1}^{\prime}\right) \otimes 1 w^{*}=p^{\prime} \mathfrak{B}\left(\mathfrak{E}_{1}\right) p^{\prime} \otimes 1$ is fulfilled. Therefore, in putting $v=u_{1}^{*} w u_{2} \in \mathfrak{B}(\mathfrak{S})$, and using $w^{*} p^{\prime} \otimes 1=w^{*}$, we easily see from the facts above that $F=v^{*} \mathfrak{M C}_{N} v$, with $v^{*} v=1$, and $v v^{*}=p \in \mathfrak{M C}_{N}$. Let $T$ be defined by $T(x)=v^{*} x v, \forall x \in \mathfrak{M}_{N}$. Now, the map $T^{\prime}: p \mathfrak{M}_{N} p \rightarrow F$, with $T^{\prime}(x)=T(x)$ for all $x \in p \mathfrak{M}_{N} p$, is a ${ }^{*}$-isomorphism between $p \mathfrak{M}_{N} p$ and $F$. In fact, $T^{\prime}$ is linear, 
and $T^{\prime}(a b)=v^{*} a b v=v^{*} a p b v=v^{*} a v v^{*} b v=T^{\prime}(a) T^{\prime}(b)$ for all $a, b$ in $p \mathfrak{M E} \varepsilon_{N} p$, and $T^{\prime}\left(a^{*}\right)=T^{\prime}(a)^{*}$ trivially. Moreover, $c \in p \mathfrak{M}_{N} p$ and $T^{\prime}(c)=0$ implies $p c p=0$, by $v v^{*}=p$, i.e. $c=0$.

Let $J=T^{\prime^{-1}}$, and $E_{F}$ a normal conditional expectation from $\mathfrak{M}$ onto $F$, and let $g \in S_{0}(\mathfrak{M})$ fixed. Let us define a map $R$ acting from $\mathfrak{M}$ into $\mathfrak{M}_{N}$ as

$$
R=J \circ E_{F}+g(\circ) p^{\perp} .
$$

Then, $R$ is normal and completely positive. Since $J(1)=p$, and $g$ is a state, $R(1)=1$ follows, i.e. $R \in C P_{1}^{0}(\mathfrak{M})$. By (5-4), for $x \in F$ we see:

$$
T \circ R(x)=v^{*} J(x) v+g(x) v^{*} p^{\perp} v=v^{*} J(x) v=T^{\prime} \circ J(x)=x .
$$

Therefore, if we define $y_{k}=R\left(x_{k}\right)$, for $k=1,2, \cdots, n$, then $\{y\}$ is a $n$-tuple of non-negative elements in $\mathfrak{M E}_{N}$ such that $T\left(y_{k}\right)=x_{k}$ for all $k$. Both maps $T, R$ are in $C P_{1}^{0}(\mathfrak{M})$. Hence

$$
\begin{aligned}
& K_{n}(\{\omega\} ;\{x\})=\sup _{s} \sum_{k} \omega_{k} \cdot S \cdot T\left(y_{k}\right) \leq K_{n}(\{\omega\} ;\{y\}) \\
& K_{n}(\{\omega\} ;\{y\})=\sup _{s} \sum_{k} \omega_{k} \cdot S \cdot R\left(x_{k}\right) \leq K_{n}(\{\omega\} ;\{x\}),
\end{aligned}
$$

with $S$ extending over $C P_{1}^{0}(\mathfrak{M})$ ). Taking together these inequalities gives

$$
K_{n}(\{\omega\} ;\{y\})=K_{n}(\{\omega\} ;\{x\})
$$

for all $n$-tuples $\{\omega\} \subset S_{0}(\mathfrak{M})$ ). By Proposition 2 (cf. (4-5)) this gives

$$
K_{n}(\{\omega\} ;\{x\})=K_{n}^{\prime}(\{\omega\} ;\{y\}) .
$$

Since $F$ is a finite dimensional subfactor of $\mathfrak{M}$, with $\mathfrak{M}$ properly infinite, we may apply the result of [17, Proposition 2.1] to see that for each given $V \in C P_{\mathrm{I}}^{0}(\mathfrak{M})$ there is an $a \in \mathfrak{M}$, with $a^{*} a=1$, such that $V(x)=a^{*} x a$ for all $x \in \mathbb{F}$. Therefore

$$
\left.K_{n}(\{\omega\} ;\{x\})=\sup \left\{\sum_{k} \omega_{k}\left(a^{*} x_{k} a\right): a^{*} G=1, a \in \mathfrak{M}\right\}\right\} .
$$

Since $\mathfrak{M}$ is properly infinite, every isometry in $\mathfrak{M}$ can be strongly approximated by unitaries. Hence, (5-8) means that $K_{n}(\{\omega\} ;\{x\})=f(\{\omega\})$. The latter, together with (5-7), proves the assertion.

Assume, $E_{N}: \mathfrak{M} \rightarrow \mathfrak{M R}_{N}$ is a normal conditional expectation from $\mathfrak{M}$ onto $\mathfrak{M}_{N}$. Let us investigate what happens to $f\left(\left\{\omega \circ E_{N}\right\}\right), f \in \mathfrak{\Im}_{n}^{+}(\mathfrak{M})$, when $N \rightarrow \infty$ is considered.

Lemma 2. For every $n$-tuple $\{\omega\} \subset S_{0}(\mathfrak{M})$ the following is true:

$$
f(\{\omega\})=\lim _{N \rightarrow \infty} f\left(\left\{\omega \circ E_{N}\right\}\right)=\sup _{N} f\left(\left\{\omega \circ E_{N}\right\}\right),
$$


$\forall f \in \mathfrak{S}_{n}^{+}(\mathfrak{M})$.

Proof. For given $f$, by Lemma $1, \exists\{y\} \subset \mathfrak{A}_{0+}$ with

$$
f(\{\nu\})=K_{n}^{\prime}(\{\nu\},\{y\}), \quad \forall\{\nu\} \subset S_{0}(\mathfrak{M} \mathcal{R}) .
$$

We know $E_{N} \in C P_{1}^{0}(\mathfrak{M})$, and $E_{N} \circ E_{M}=E_{M}$, for $N \geq M$. Hence, (5-10) allows us to apply Theorem 1 , especially $(3) \Leftrightarrow(5)$, twice with the result

$$
f\left(\left\{\omega \circ E_{M}\right\}\right)=f\left(\left\{\omega \circ E_{N} \circ E_{M}\right\}\right) \leq f\left(\left\{\omega \circ E_{N}\right\}\right) \leq f(\{\omega\}),
$$

which proves the existence of the expression on the right hand side of (5-9). Let $\varepsilon>0$. Then, to each $\{\omega\}$ of $S_{0}(\mathfrak{M})$, an subscript $N_{0}$ is easily found such that

$$
\exists u \in \mathcal{U}\left(\mathfrak{M}_{N_{0}}\right) \Rightarrow K_{n}^{\prime}(\{\omega\},\{y\})-\varepsilon \leq \sum \omega_{k}\left(u^{*} y_{k} u\right) .
$$

Indeed, Proposition 2, (4-5) makes that this is possible. Let $N_{1}$ such that $\{y\} \subset \mathfrak{M i}_{N_{1}}$. If $N \geq \max \left\{N_{0}, N_{1}\right\}$, we have $E_{N}\left(u^{*} y_{k} u\right)=u^{*} y_{k} u, \forall k$, for, $u^{*} y_{k} u \in$ $\mathfrak{M E}_{N}$ holds and $E_{N}$ projects onto $\mathfrak{M E}_{N}$. Therefore,

$$
\sum \omega_{k}^{u}\left(y_{k}\right) \leq K_{n}^{\prime}\left(\left\{\omega \circ E_{N}\right\},\{y\}\right)=f\left(\left\{\omega \circ E_{N}\right\}\right),
$$

for all $N \geq \max \left\{N_{0}, N_{1}\right\}$, where we have been using the representation (5-10). Taking together (5-12), (5-13), and making use of (5-10) once more again, yields

$$
f(\{\omega\})-\varepsilon \leq f\left(\left\{\omega \circ E_{N}\right\}\right),
$$

for $N$ sufficiently large. Since $\varepsilon>0$ could have been chosen at will, and the information of (5-11) is available, (5-14) implies (5-9).

Lemma 3. Let $f_{\{x\}} \in \mathfrak{Y}_{n}^{+}(\mathfrak{M})$ be generated by $\{x\} \in \Lambda_{n}(\mathfrak{M})_{+}$. Then,

$$
\left\|\sum x_{k}\right\| \leq f_{\{x\}} \leq \Sigma\left\|x_{k}\right\| \text {, over the n-tuples, }
$$

moreover, the following equivalences take place:

$$
f_{\{x\}}(\{\omega\})=\left\|\sum x_{k}\right\|, \forall\{x\} \in \Lambda_{n}(\mathfrak{M})_{+}, \Leftrightarrow
$$

$\{\omega\}$ is an orthogonal system of states:

$$
f_{\{x\}}(\{\omega\})=\left\|\sum x_{k}\right\|, \forall\{x\} \in \Lambda_{n}(\mathfrak{M})_{+}, \Leftrightarrow \omega_{1}=\cdots=\omega_{n} .
$$

Proof. The right hand part of the estimation in (5-15) is obviously valid. To see the other one, let us look on the linear map $T$ given by $T(x)=\nu(x) 1$, with an arbitrarily chosen $\nu \in S_{0}(\mathfrak{M})$. Then, $\omega_{k} \circ T=\nu, \forall k$. By Lemma 1, 
$\exists\{y\} \subset \mathfrak{A}_{0+}$ such that (we suppose $f$ is generated by $\{x\}$ )

$$
f(\{\mu\})=K_{n}^{\prime}(\{\mu\},\{y\}), \forall\{\mu\} \subset S_{0}(\mathfrak{M}) .
$$

Since $T$ is unital and completely positive, normal, Theorem 1 says that for $\{\nu\}=\{\nu, \cdots, \nu\}$

$$
f(\{\nu\}) \leq f(\{\omega\}) \text { 。 }
$$

But then, $f(\{\nu\})=\sup _{u \in \mathcal{U}(\mathfrak{M})} \nu^{u}\left(\sum x_{k}\right) \leq\left\|\sum x_{k}\right\|$. To see equality to hold, let $p \in \mathfrak{M}, p \neq \mathcal{O}$, an orthoprojection with $p\left(\sum x_{k}\right) p=\left\|\sum x_{k}\right\| p$. Since $\{x\} \subset \mathfrak{U}_{0+}$, such $p$ exists. Let us now for $\nu$ specify a normal state with support on $p$, i.e. $\nu(p)=1$. Then, by the previously clarified things, $\nu\left(\sum x_{k}\right)=f(\{\nu\})=\left\|\sum x_{k}\right\|$. Together with (5-19) we get the left hand side estimation of (5-15). Note that, when taking $T(x)=\omega(x) 1, \forall x \in \mathfrak{M}$ for an arbitrary other normal state $\omega$, by the same as above reasoning with Theorem 1 we see $f(\{\omega, \cdots, \omega\}) \leq f(\{\nu, \cdots, \nu\})=$ $\left\|\sum x_{k}\right\|$, which together with (5-15) (which has been proved above) implies the back-part of (5-17) to be true. On the other hand, assume $f_{i x\}}(\{\omega\})=\left\|\sum x_{k}\right\|$, $\forall\{x\} \in \Lambda_{n}(\mathfrak{M})_{+}$. Because of (5-18) and (5-15), (5) of Theorem 1 happens to be true for any other $n$-tuple $\{\sigma\} \subset S_{0}(\mathfrak{M})$. Especially, the $n$-tuple $\{\nu, \cdots, \nu\}$ might be chosen. Theorem 1 then asserts that $\Phi \in S T\left(\mathfrak{M}_{*}\right)$ exists with $\omega_{k}=$ $\Phi(\nu), \forall k$, which obviously can only be true if $\omega_{1}=\cdots=\omega_{n}$. This proves the implication $\Rightarrow$ of (5-17).

To see (5-16), assume the left hand part to hold. Then, (5-15) together with (5-18) and Theorem 1 implies for each other $n$-tuple $\{\nu\} \subset S_{0}(\mathfrak{M})$ the existence of $T \in C P_{1}^{0}(\mathfrak{M})$ with $\nu_{k}=\omega_{k} \circ T, \forall k$. Hence, $\left\|\nu_{k}-\nu_{j}\right\|_{1}=\left\|\left(\omega_{k}-\omega_{j}\right) \circ T\right\|_{1} \leq$ $\left\|\omega_{k}-\omega_{j}\right\|_{1}, \forall k, j$. Since the $n$-tuple $\{\nu\}$ might be chosen at will, assume $\{\nu\}$ is a system of mutually orthogonal, normal states (since $\mathfrak{M}$ is infinite dimensional, to every $n$ such a system can be chosen). For $k \neq j$ we have $\left\|\nu_{k}-\nu_{j}\right\|_{1}=2$. Therefore, by the inequality above, $\left\|\omega_{k}-\omega_{j}\right\|_{1}=2, \forall k \neq j$, i.e. $\{\omega\}$ is an orthogonal system of states. This proves the $\Rightarrow$-implication of (5-16).

To see the other way around, assume $\{\nu\}$ is a $n$-tupel of mutually orthogonal normal states over $\mathfrak{M}$. Let us denote by $s_{1}, \cdots, s_{n}$ the support projections of the $\nu_{k}$ 's. To each $n$-tuple $\{\omega\}$ of normal states let us associate a linear map $\Phi: \mathfrak{M}_{*} \rightarrow \mathfrak{M}_{*}$ defined through the settings

$$
\Phi(\rho)=\sum_{j \leq n-1} \rho\left(s_{j}\right) \omega_{j}+\rho\left(1-\sum_{j \leq n-1} s_{j}\right) \omega_{n}, \quad \forall \rho \in \mathfrak{M}_{*} .
$$

By construction, $\Phi \in S T\left(\mathfrak{M}_{*}\right)$. Therefore, Theorem 1 applies and yields

$$
K_{n}^{\prime}(\{\omega\},\{z\}) \leq K_{n}^{\prime}(\{\nu\},\{z\}), \quad \forall\{\omega\} \subset S_{0}(\mathfrak{M} \mathcal{\mathcal { C }}),
$$


for every $\{z\} \subset \mathfrak{A}_{0+}$. Given such a $\{z\}$, we can provide us with orthoprojections $p_{1}, \cdots, p_{n}$ such that $p_{j} z_{j} p_{j}=\left\|z_{j}\right\| p_{j}, \forall j$. Since $\{z\} \subset \mathbb{M}_{N}$ for a suitable subscript $N$, all $p_{k}$ may be supposed dimensionally infinite (with respect to $\mathfrak{B}(\mathfrak{E})$ ). Then, a result of [19, 4.3 Lemma] applies and gives the existence of a $n$-tuple $\{\xi\} \subset \mathfrak{S}$ of mutually orthogonal unit vectors $\xi_{k}$ in $\mathfrak{S}$ such that $p_{k} \xi_{k}=\xi_{k}$, $\forall k$. Defining $\omega_{k}(x)=\left\langle\xi_{k}, x \xi_{k}\right\rangle, \forall x \in \mathfrak{M}$, i.e. the vector state over $\mathfrak{M}$ generated by $\xi_{k}$, we see that $\omega_{k}\left(z_{k}\right)=\omega_{k}\left(p_{k} z_{k} p_{k}\right)=\left\|z_{k}\right\|, \forall k$, by assumptions on the choice of the $p_{k}$ 's. By (5-15), we have to follow that $K_{n}^{\prime}(\{\omega\},\{z\})=\sum\left\|z_{k}\right\|_{\text {. Owing }}$ to (5-20), and using (5-15) once more again, we see

$$
K_{n}^{\prime}(\{\nu\},\{z\})=\sum\left\|z_{k}\right\| \text {. }
$$

The latter holds for each $\{z\} \subset \mathfrak{Q}_{0+}$. Suppose $\{x\} \in A_{n}(\mathfrak{M})_{+}$. Then, (5-18) reads as

$$
f_{\{x\}}(\{\nu\})=K_{n}^{\prime}(\{\nu\},\{y\}), \text { with } \quad y_{k}=R\left(x_{k}\right) \in \mathfrak{A}_{0+}, \text { Rc.p., unital }
$$

where Lemma 1 and the special form of the $y_{k}$ from the proof there has been referred to. Since also $x_{k}=v^{*} y_{k} v, v \in \mathfrak{M}_{1}$, we see $\left\|y_{k}\right\|=\left\|x_{k}\right\|, \forall k$, and (5-21), (5-22) imply the desired equality. This closes the proof.

\section{$\S$ 6. The Main Results}

Theorem 2. Let $\mathfrak{M}$ be a non-finite AFD-factor over some separable Hilbertspace $\mathfrak{S}$. Let $\{\omega\},\{\sigma\} \subset S_{0}(\mathfrak{M})$ be $n$-tuples of normal states. The following conditions are equivalent to each other:

(1) $\{\omega\} \gg\{\sigma\}$, i.e. $\exists \Phi \in S T\left(\mathfrak{M} *^{*}\right)$ with $\omega_{k}=\Phi\left(\sigma_{k}\right), \forall k$;

(2) $\{\omega\} \gg_{*}\{\sigma\}$, i.e. $\exists \Phi \in S T\left(\mathfrak{M}_{*}\right)$ with $\omega_{k}=\Phi\left(\sigma_{k}\right), \forall k$;

(3) $\exists T \in C P_{1}(\mathfrak{M})$ with $\omega_{k}=\sigma_{k} \circ T, \forall k$;

(4) $\exists T \in C P_{1}^{0}(\mathfrak{M})$ with $\omega_{k}=\sigma_{k} \circ T, \forall k$;

(5) $f(\{\omega\}) \leq f(\{\sigma\})$ for all $f \in \mathfrak{Y}_{n}^{+}(\mathfrak{M})$;

(6) $g(\{\omega\}) \geq g(\{\sigma\})$ for all $g \in \mathfrak{J}_{n}^{-}(\mathfrak{M})$.

Thus, $\mathfrak{\Im}_{n}^{-}(\mathfrak{M})$ and $\mathfrak{\Im}_{n}^{+}(\mathfrak{M})$ provide sufficient systems of $\gg$-isotonic and $\gg$-antiisotonic functions, respectively.

Proof. Follows at once from Theorem 1 and Lemma 1. The latter telling us that $\mathfrak{\Im}(\mathfrak{A})=\mathfrak{F}_{n}^{+}(\mathfrak{M})$, for every generating U.H.F.- $C^{*}$-subalgebra $\mathfrak{A}$ of $\mathfrak{M}$. The equivalence $(5) \Leftrightarrow(6)$ is due to $(5-1),(5-2)$.

Remark. Note that Lemma 2, Lemma 3 have their counterparts in case of $\mathfrak{F}_{n}^{-}(\mathfrak{M})$. By Lemma $1, \mathfrak{S}(\mathfrak{U})=\mathfrak{F}_{n}^{+}(\mathfrak{M})$ for any generating U.H.F.- $C^{*}$-sub- 
algebra $\mathfrak{A}$ of $\mathfrak{M}$. Hence, we arrived at an intrinsic characterization for a particular family of $\gg$-isotonic resp. anti-isotonic functions without to have need to refer to the (non-unique) substructure $\mathfrak{2}$.

For convenience, let us list up some of the properties of the functions collected into $\mathfrak{\Im}_{n}^{+}(\mathfrak{M})$ (an analogous dual list hold in case of $\mathfrak{\Im}_{n}^{-}(\mathfrak{M} \mathcal{M})$ ). Let $f \in \mathfrak{Y}_{u}^{+}(\mathfrak{M})$. Then:

$f$ is uniformly continuous,

$f$ is (relatively) $w^{*}$-lower semicontinuous,

$f$ is (simultaneously) $\mathcal{U}(\mathfrak{M})$-invariant,

i.e. $f\left(\left\{\omega^{u}\right\}\right)=f(\{\omega\})$, with $\left\{\omega^{u}\right\}=\left\{\omega_{1}^{u}, \cdots, \omega_{n}^{u}\right\}$,

$f$ is (jointly) convex

over the set of all n-tuples of normal states on $\mathfrak{M}$. Moreover, if $f_{\{x\}}$ is generated by the a.f.d.-system $\{x\}$, then

$$
\left\|\sum x_{k}\right\| \leq f_{\{x\}} \leq \sum\left\|x_{k}\right\|
$$

and

$$
f_{\{x\}}(\{\omega\})=\sum\left\|x_{k}\right\|, \forall\{x\} \in \Lambda_{n}(\mathfrak{M})_{+}, \Leftrightarrow\{\omega\}
$$

is an orthogonal system of normal states;

$$
f_{\{x\}}(\{\omega\})=\Sigma\left\|x_{k}\right\|, \forall\{x\} \in \Lambda_{n}(\mathfrak{M} \mathcal{M})_{+}, \Leftrightarrow \omega_{1}=\cdots=\omega_{n} .
$$

Furthermore, each $f \in \mathfrak{S}_{n}^{+}(\mathfrak{M})$ has the isotony-property, i.e. if $\mathfrak{M}_{1} \subset \mathfrak{M} \mathfrak{R}_{2} \subset \cdots \subset \mathfrak{M}$ is a sequence of finite-dimensional subfactors of $\mathfrak{M}$ which generates $\mathfrak{M}$, and $E_{N} ; \mathfrak{M} \rightarrow \mathfrak{M}_{N}$ is a sequence of associated normal conditional expectations, then

$$
f\left(\left\{\omega \circ E_{1}\right\}\right) \leq f\left(\left\{\omega \circ E_{2}\right\}\right) \leq \cdots \leq f(\{\omega\})
$$

and

$$
\lim _{N \rightarrow \infty} f\left(\left\{\omega \circ E_{N}\right\}\right)=f(\{\omega\}) .
$$

These properties are either obvious, or they are collected together from all we have been deriving in the previous sections.

\section{§7. The Case of the Admissible vN-Algebras}

Let $\mathfrak{N}$ be a vN-algebra that is admissible in our sense (see Section 2). Thus, there is a properly infinite AFD-factor $\mathfrak{M}$ which contains $\mathfrak{N}$, and there is a normal conditional expectation $E$ projecting from $\mathfrak{M}$ onto $\mathfrak{N}$. In these notations, the result of [18, Proposition] and our Theorem 2 imply the following to be valid: 
Corollary. For n-tuples $\{\omega\},\{\sigma\} \subset S_{0}(\mathfrak{N})$ the subsequently listed conditions are mutually equivalent

(1) $\{\omega\} \gg\{\sigma\}$;

(2) $\{\omega\} \gg_{*}\{\sigma\}$;

(3) $f(\{\omega \circ E\}) \leq f(\{\sigma \circ E\})$ for all $f \in \mathfrak{S}_{n}^{+}(\mathfrak{M})$; (!)

(4) $g(\{\omega \circ E\}) \geq g(\{\sigma \circ E\})$, for all $g \in \mathfrak{F}_{n}^{-}(\mathfrak{M})$. (!)

Therefore, the set of functions $\left\{f(\{(\cdot) \circ E\}): f \in \mathfrak{S}_{n}^{-}(\mathfrak{M})\right\}$ forms, e.g., a sufficient family of $\gg$-isotonic functions.

As an application, let us consider the case $\mathfrak{M}=\mathfrak{B}(\mathfrak{S})$, and $\mathfrak{N} \simeq l^{\infty}$. Let $\left\{\xi_{j}: j \in N\right\}$ be a complete orthonormal system in $\mathfrak{L}$, and denote by $q_{j}$ the orthoprojection onto $\left[\xi_{j}\right]$. We might assume that $\mathfrak{N}$ is generated by the $q_{j}$ 's. Then, $E(\cdot)=\sum q_{j}(\cdot) q_{j}$ is the normal conditional expectation onto $\mathfrak{N}$. Let $\{x\} \in \Lambda_{n}(\mathfrak{B}(\mathfrak{S}))_{+} . \quad$ Let $\{\omega\} \subset S_{0}(\mathfrak{N})$. We find non-negative reals $\omega^{k 1}$, with $\sum_{1} \omega^{k 1}=1, \forall k$, such that $\omega_{k} \circ E=\sum_{1} \omega^{k 1} q_{1}$. Therefore, we have $f_{(x)}(\{\omega \circ E\})=$ $\sup _{u} \sum \omega_{k} \circ E\left(u^{*} x_{k} u\right)=\sup _{u} \sum_{1}\left\langle u \xi_{l}, y_{l} u \xi_{l}\right\rangle=f_{\{y\}}(\{\nu\})$, with $y_{l}=\sum_{k} \omega^{k l} x_{k}, \nu_{k}\left({ }^{\circ}\right)=$ $\left\langle\xi_{k},(\cdot) \xi_{k}\right\rangle$. The system $\{\nu\}$ being an orthogonal one, and since $\sum\left\|y_{1}\right\|<\infty$, makes that (6-6) (with a little modification) applies with the result:

$$
f_{\{x\}}(\{\omega \circ E\})=\sum_{l}\left\|y_{l}\right\|=\sum_{l}\left\|\sum_{k} \omega^{k l} x_{k}\right\| .
$$

This, however, gives Example 5 in case $\Omega=\mathbb{N}$, $\mu$-the counting measure, essentially (compare this to the sufficient system given at the end of Section 2).

\section{Acknowledgements}

This work has been prepared while the author was staying at the ETH Zürich in winter of $1985 / 86$. Thanks to Prof. H. Primas and his group, Laboratorium für Physikalische Chemie der ETH, for the warm hospitality during the stay, and many interesting discussions. Last but, not least, I am deeply indepted to Prof. Dr. A. Uhlmann, Sektion Physik der Karl-Marx-Universitat Leipzig, for taking steadily and constructively interest in the progress of this research.

\section{References}

[1] Marshall, A.W. and Olkin, I., Inequalities: Theory of Majorization and Its Applications, Academic Press, New-York-London-Toronto-Sydney-San Francisco, (1979).

[2] Sakai, S., $C^{*}$-clgebras and $W^{*}$-algetras, Springer-Verlag, Berlin-Heidelberg-NY, (1971).

[3] Takesaki, M., Theory of operator algebras I, Springer Verlag, NY-Heidelberg-Berlin, (1979). 
[4] Schaefer, H.H., Banach Lattices and Positive Operators, Springer-Verlag, Berlin-Heidelberg-NY, (1974).

[5] Hardy, G.H., Littlewood, J.E. and Polya, G., Inequalities, Cambridge University Press, New York, (1934).

[6] Alberti, P.M. and Uhlmann, A., Stochasticity and Partial Order (Doubly Stochastic Maps and Unitary Mixing), D. Reidel Publ. Comp., Dordrecht, (1982).

[7] Uhlmann, A., Endlichdimensionale Dichtematrizen I, II, Wiss. Z. KMU Leipzig, 21, 421 (1972), 22 (1973), 139.

[8] Thirring, W., Lehrbuch der Mathematischen Physik, Bd. 4, Springer-Verlag, Wien-New York, (1980).

[9] Wehrl, A., General properties of entropy, Rev. Mod. Phys., 50 (1978), 81.

[10] Ruch, E., Schranner, R. and Seligman, T.H., Generalization of a Theorem by Hardy, Littlewood, and Polya, J. Math. Anal. Appl., 76, No. 1 (1980), 222-229.

[11] Alberti, P.M. and Uhlmann, A., A problem relating positive maps on matrix algebras, Rep. Math. Phys., 18, 2 (1980), 163.

[12] - Existence and Density Theorems for Stochastic Maps on Commutative $C^{*}$-algebras, Math. Nachr., 97 (1980), 279-295.

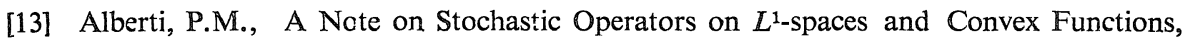
J. Math. Anal. Appl., to appear.

[14] Alberti, P.M., Uhlmann, A., Dissipative Motion in State Spaces, Teubner-Texte zur Mathematik, Bd. 33, Teubner-Verlag, Leipzig, (1981).

[15] Ando, T., Majorization, Doubly Stochastic Matrices and Comparison of Eigenvalues, Sapporo, (1982).

[16] Connes, A., Classification of injective factors, Ann. Math., 104 (1976), 73-115.

[17] Haagerup, U., A New Proof of the Equivalence of Injectivity and Hyperfiniteness for Factors on a Separable Hilbertspace, Journ. Funct. Analysis, 62, No. 2 (1985), 160-200.

[18] Alberti, P.M., Completely positive stochastic linear maps over AFD-factors and unitary maixing on generating U.H.F.-subalgebras, preprint (1985), Publ. RIMS Kyoto Univ., $22.12 .86(1986)$.

[19] - On the simultaneous transformation of density operators by means of a c.p., unity preserving linear map, Publ. RIMS Kyoto Univ., 2il, No. 3 (1985), 619-644. 
